\title{
CONVERGENT TRANSFER SCHEMES FOR $N$-PERSON GAMES
}

\author{
R. E. STEARNS
}

Introduction. The object of this paper is to describe a transfer scheme that converges to the kernel of a game and another scheme that conver ges to the bargaining set.

Both the bargaining set and the kernel can be described as payoff vectors in which every objection (appropriately defined for each case) by one player against another player has a counter-objection. If, for a given payoff vector, a player $i$ can sustain an objection against another player $j$, a reasonable negotiation step might be for $j$ to transfer enough of his payoff to $i$ so that $i$ can no longer sustain his objection. This results in a new payoff vector subject to further negotiation. We will show that any infinite sequence of such transfers causes the payoff vector to converge to a vector in the appropriate bargaining set provided only that maximal transfers are frequently made. Thus negotiation can approach the bargaining set without the players solving the defining conjunctive-disjunctive system of linear inequalities. Also, this scheme can be used as a computational technique to find one and possibly more bargaining points. The desired convergence is established by proving the convergence of a general transfer scheme of which the kernel and bargaining set schemes are special cases.

For the purpose of this paper, a game is given by an ordered pair $(N, v)$ where $N$ is an $n$-element set of players and $v$, the characteristic function, is a zero-one normalized set function over the subsets of $N$ (i.e. $v$ satisfies $v(\{i\})=0$ for all $i$ in $N$ and $v(N)=1$ ).

Let $E$ be the space of real $n$-vectors indexed by $N$ and let $A$ be the set of all group and individually rational payoff vectors. Under the zero-one normalization, $A$ is the set of $x$ in $E$ such that

$$
\sum_{N} x_{l}=1 \text { and } x_{i} \geqq 0 \quad \text { for all } i \text { in } N .
$$

For $S \subseteq N$ and $x$ in $E$, we define

$$
e(S, x)=v(S)-\sum_{S} x_{l},
$$

the excess of $S$ in $x$. For distinct players $i$ and $j$ and for all $x$ in $E$, define

$$
s_{i j}(x)=\max \{e(S, x) \mid\{i\} \subseteq S \subseteq N-\{j\}\}
$$

Received by the editors July 21, 1967. 
the maximum excess of $i$ over $j$. Intuitively, $s_{i j}(x)$ represents the potential payoff units which player $i$ can offer the members of some coalition $S$ to act for their own advantage against the interest of player $j$. This quantity plays a key role in what follows.

Transfers and $K$-transfers. If $x$ is in $A, a$ and $b$ are distinct players in $N$, and $\alpha \geqq 0$, then $y$ in $A$ is said to result from $x$ by a transfer of size $\alpha$ from $b$ to $a$ if and only if

$$
\begin{aligned}
& y_{a}=x_{a}+\alpha, \\
& y_{b}=x_{b}-\alpha, \\
& y_{i}=x_{i} \quad \text { otherwise. }
\end{aligned}
$$

We say that $y$ is the result of a $K$-transfer of size $\alpha$ if, in addition to (4), (5), and (6), also

$$
s_{a b}(y) \geqq s_{b a}(y) .
$$

LEMMA 1. If $y$ results from $x$ by a transfer of size $\alpha$ from $b$ to $a$, then

$$
\begin{aligned}
& s_{a b}(y)=s_{a b}(x)-\alpha, \\
& s_{b a}(y)=s_{b a}(x)+\alpha .
\end{aligned}
$$

Proof. For all $S,\{a\} \subseteq S \subseteq N-\{b\}$, equations (2), (4), and (6) give

$$
e(S, y)=v(S)-\sum_{S-a} x_{i}-x_{a}-\alpha=e(S, x)-\alpha .
$$

Since each excess of $a$ over $b$ is reduced by $\alpha$, so must the maximum excess given in (3) and thus (8) is proved. A very similar argument proves (9).

From Lemma 1 and (7), we see that a $K$-transfer from $b$ to $a$ may be described as transfer which reduces the difference $s_{a b}(x)-s_{b a}(x)$ without making it negative.

LEMMA 2. If $y$ results from $x$ by $a K$-transfer $\alpha$ from $b$ to $a$, then for all distinct $i$ and $j$,

$$
s_{i j}(y)>s_{a b}(y) \quad \text { implies } \quad s_{i j}(x) \geqq s_{i j}(y) \text {. }
$$

Proof. Let $S$ be a set such that $\{i\} \subseteq S \subseteq N-\{j\}$ and $e(S, y)=s_{i j}(y)$. If $b$ is in $S$, then $a$ must be in $S$, for otherwise we would have from (3) that

$$
s_{b a}(y) \geqq e(S, y)=s_{i j}(y)>s_{a b}(y)
$$

which contradicts (7). Therefore (4), (5), and (6) imply $\sum_{s}\left(y_{l}-x_{l}\right) \geqq 0$ since the only possible negative term in the sum would be $y_{b}-x_{b}=-\alpha$ in which case the sum would also contain the compensating term $y_{a}-x_{a}=\alpha$. Therefore $\sum_{s} y_{l}>\sum_{s} x_{l}$ which implies $e(S, x) \geqq e(S, y)$ and we get

which is (10).

$$
s_{i j}(x) \geqq e(S, x) \geqq e(S, y)=s_{i j}(y)
$$


Thus a $K$-transfer cannot increase the larger values of $s_{i j}$. An examination of this constraint in more detail will give the bounds that ensure convergence. To this end, we now introduce some new notation.

For each $x$ in $E$ and $1 \leqq k \leqq n(n-1)$, let $H_{k}(x)$ be a $k$ element set of ordered distinct pairs on $N$ such that $s_{i j}(x) \geqq s_{i^{\prime} j^{\prime}}(x)$ for all $(i j)$ in $H_{k}(x)$ and all $\left(i^{\prime} j^{\prime}\right)$ not in $H_{k}(x)$. Let

$$
\varphi_{k}(x)=\sum_{H_{k}(x)} s_{i j}(x)
$$

In other words, $\varphi_{k}(x)$ is the sum of the $k$ largest $s_{i j}(x)$.

LEMMA 3. If $x$ and $y$ are in $A$, then

$$
\left|\varphi_{k}(y)-\varphi_{k}(x)\right| \leqq k .
$$

If $y$ results from $x$ by a transfer of size $\alpha$, then

$$
\left|\varphi_{k}(y)-\varphi_{k}(x)\right| \leqq k \cdot \alpha .
$$

Proof. Because $x$ and $y$ are in $A$, we know from (1) that for any $S,\left|\sum_{s} x_{l}-\Sigma_{s} y_{l}\right|$ $\leqq 1$. This implies that $e(S, x) \geqq e(S, y)-1$ and consequently $s_{i j}(x) \geqq s_{i j}(y)-1$ for all distinct pairs $i$ and $j$. Using this last inequality and the definition of $H_{k}$, we obtain

$$
\varphi_{k}(x)=\sum_{H_{k}(x)} s_{i j}(x) \geqq \sum_{H_{k}(y)} s_{i j}(x) \geqq \sum_{H_{k}(y)}\left(s_{i j}(y)-1\right)=\varphi_{k}(y)-k
$$

which is equivalent to $\varphi_{k}(y)-\varphi_{k}(x) \leqq k$ and this inequality together with the symmetry between $x$ and $y$ gives (12). In case where $y$ results from a transfer of size $\alpha$, equations (4), (5) and (6) give $\left|\sum_{s} x_{l}-\sum_{s} y_{l}\right| \leqq \alpha$ and the same chain of reasoning gives (13).

LEMMA 4. If $y$ results from $x$ by a K-transfer of size $\alpha$, then there is a $\bar{k}$ such that

$$
\begin{aligned}
& \varphi_{\bar{k}}(y)-\varphi_{\bar{k}}(x) \leqq-\alpha, \\
& \varphi_{k}(y)-\varphi_{k}(x) \leqq 0 \quad \text { for all } k \leqq \bar{k}
\end{aligned}
$$

Proof. Let the $K$-transfer be from player $b$ to player $a$. Let $\bar{k}$ be the number of ordered pairs $(i j)$ such that $s_{i j}(x) \geqq s_{a b}(y)$. Clearly $\bar{k} \geqq 1$ by (8). Let $k$ satisfy $k \leqq \bar{k}$, and let

Observe that

$$
l=\min _{H_{k}(x)}\left\{s_{i j}(x)\right\}
$$

$$
l \geqq s_{a b}(y)
$$

because of the choice of $\bar{k}$. Since $H_{k}(x)$ and $H_{k}(y)$ are $k$ element sets, there is a one-to-one correspondence between these sets such that pair (ij) corresponds to 
itself for all (ij) in $H_{K}(x) \cap H_{k}(y)$. Let $(i j)$ be an arbitrary element of $H_{k}(y)$ and $\psi(i j)$ the corresponding pair in $H_{k}(x)$. If (case 1$) s_{i j}(y) \leqq l$, then automatically

$$
s_{i j}(y) \leqq s_{\psi(i j)}(x)
$$

by choice of $l$. If (case 2) $s_{i j}(y)>l$, then (16) and (10) together give $s_{i j}(x) \geqq s_{i j}(y)>l$. This implies (ij) is in $H_{k}(x)$ which implies $\psi(i j)=(i j)$ and (17) again is true. Thus (17) is always true and it follows that

$$
\varphi_{k}(y)-\varphi_{k}(x)=\sum_{H_{k}(y)}\left(s_{i j}(y)-s_{\psi(i j)}(x)\right) \leqq 0
$$

which is (15).

In the special case $k=\bar{k},(a b)$ is in $H_{k}(x)$ by (8) and the definition of $\bar{k}$. If (case 1) $\psi^{-1}(a b)=a b$ then equation (8) gives

$$
s_{\psi}-1_{(a b)}(y) \leqq s_{a b}(x)-\alpha .
$$

If (case 2) $\psi^{-1}(a b)=\left(a^{\prime} b^{\prime}\right) \neq(a b)$ then $\psi\left(a^{\prime} b^{\prime}\right) \neq\left(a^{\prime} b^{\prime}\right),\left(a^{\prime} b^{\prime}\right)$ is not in $H_{k}(x)$, and $s_{a^{\prime} b^{\prime}}(x)<s_{a b}(y)$ by definition of $\bar{k}$. Because of $(10), s_{a^{\prime} b^{\prime}}(y)>s_{a b}(y)>s_{a^{\prime} b^{\prime}}(x)$ is impossible and so we must also have $s_{a^{\prime} b^{\prime}}(y) \leqq s_{a b}(y)$. This inequality with (8) gives (19). Thus in either case, (19) holds and the $\psi^{-1}(a b)$ term of (18) has value $-\alpha$. Since the other terms have value $\leqq 0$ by (17), this proves (14).

Now we are ready to apply the lemmas to bounds of sequences. Assume that $\left\{x^{i}\right\}$ is a sequence of vectors for $i \geqq 0$ such that each $x^{i+1}$ results from $x^{i}$ by a $K-$ transfer of size $\alpha^{i}$. For each $r \geqq 1$, let

$$
\begin{aligned}
& I_{k}^{r}=\sum_{0}^{r-1} \max \left(0, \varphi_{k}\left(x^{i+1}\right)-\varphi_{k}\left(x^{i}\right)\right), \\
& D_{k}^{r}=-\sum_{0}^{r-1} \min \left(0, \varphi_{k}\left(x^{i+1}\right)-\varphi_{k}\left(x^{i}\right)\right) .
\end{aligned}
$$

$I_{k}^{r}$ is just the sum of the increases in the first $r$ values of $\varphi_{k}$ and $D_{k}^{r}$ is the absolute value of the sum of the decreases.

LEMMA 5. The quantities defined above satisfy the following three relationships:

$$
\begin{aligned}
I_{k}^{r} & \leqq k \cdot \sum_{1 \leqq j<k} D_{j}^{r}, \\
D_{k}^{r} & \leqq I_{k}^{r}+k, \\
\sum_{0}^{r-1} \alpha^{i} & \leqq \sum_{k=1}^{n \cdot(n-1)} D_{k}^{r} .
\end{aligned}
$$

Proof. To save space, we represent the terms of (20) and (21) by

$$
\begin{aligned}
& \delta_{k}^{+}(i)=\max \left(0, \varphi_{k}\left(x^{i+1}\right)-\varphi_{k}\left(x^{i}\right)\right), \\
& \delta^{-}(i)=-\min \left(0, \varphi_{k}\left(x^{i+1}\right)-\varphi_{k}\left(x^{i}\right)\right) .
\end{aligned}
$$


If (case 1) $\delta_{k}^{+}(i)=0$, then

$$
\delta_{k}^{+}(i) \leqq k \cdot \sum_{1 \leqq j<k} \delta_{j}^{-}(i)
$$

since the right-hand terms (if any) are always nonnegative.

If (case 2) $\delta_{k}^{+}(i)>0$, then the $\vec{k}$ of Lemma 4 (with $x=x^{i}, y=x^{i+1}$ ) satisfies $\bar{k}<k$ because the inequality of (15) fails. Inequality (14) is equivalent to the statement

$$
\delta_{\bar{k}}^{-}(i) \geqq \alpha^{i}
$$

and this together with (13) implies

$$
\delta_{k}^{+}(i) \leqq k \cdot \delta_{\bar{k}}^{-}(i) \leqq k \cdot \sum_{1 \leqq j<k} \delta_{\bar{j}}^{-}(i)
$$

Thus (25) holds in either case and adding (25) from $i$ equal 0 to $r-1$ gives (22). Since $\bar{k}$ can be no larger than $n \cdot(n-1)$, the number of distinct ordered pairs on $N$, (25a) gives

which sums to give (24).

$$
\alpha^{i} \leqq \delta_{\bar{k}}^{-}(i) \leqq \sum_{j=1}^{n \cdot(n-1)} \delta_{j}^{-}(i)
$$

To prove (23), observe that

which sums to give

$$
\delta_{k}^{-}(i)-\delta_{k}^{+}(i)=\varphi_{k}\left(x^{i}\right)-\varphi_{k}\left(x^{i+1}\right)
$$

$$
D_{k}^{r}-I_{k}^{r}=\varphi_{k}\left(x^{0}\right)-\varphi_{k}\left(x^{r}\right)
$$

and the right-hand side of the equation is less than $k$ by (12) and inequality (23) follows.

THEOREM 1. Given a set of players $N$, there exists a constant $\beta(n)$ such that for all zero-one normalized characteristic functions $v$ on $N$ and all $K$-transfer sequences $\left\{x^{i}\right\}$ on $(N, v)$ with sizes $\alpha^{i}$,

$$
\sum_{i=0}^{r} \alpha^{i} \leqq \beta
$$

for all integers $r$.

Proof. Equation (22) says that $I_{1}^{r}=0$. Therefore, by (23), $D_{1}^{r} \leqq 1$. Assuming that there are bounds for $I_{j}^{r}$ and $D_{j}^{r}$ for $j<l,(22)$ gives a bound for $I_{l}^{r}$ and then (23) gives a bound for $D_{l}^{r}$. Therefore, by induction, all the $I_{k}^{r}$ and $D_{k}^{r}$ have bounds. The derivation of these bounds has been independent of $N, v$, and $\left\{x^{i}\right\}$, except for Lemma 3 where the normalization was used (it was assumed that $A$ is given by (1)). Therefore, by (24), $\sum \alpha^{i}$ has a bound which depends only on $n$.

Demand functions. Given a game $(N, v)$, let $D=\left\{d_{i j}\right\}$ be a set of functions indexed over all ordered distinct player pairs such that each $d_{i j}$ maps $A$ into the nonnegative real numbers. A transfer of size $\alpha$ from $j$ to $i$ whereby $y$ results from $x$ is 
called a $D$-bounded transfer if and only if $\alpha \leqq d_{i j}(x)$. A member $d_{i j}$ of $D$ is called a demand function if and only if

$$
d_{i j}(x) \leqq x_{j} \text { for all } x \text { in } A .
$$

Observe that any $\alpha$ such that $x_{j}<\alpha$ is automatically disqualified as a transfer amount from $j$ to $i$ since equation (5) would require the obtained vector to be outside of $A$. On the other hand, $\alpha \leqq x_{j}$ ensures that such a transfer is possible. Thus the term "demand function" is applied to those $d_{i j}$ which guarantee there be a resulting vector in $A$; and all $d_{i j}$ in $D$ may be truncated by (27) into demand functions without changing the set of $D$-bounded transfers. For unnormalized games, (27) must be replaced by $d_{i j}(x) \leqq x_{i}-v(i)$.

For a given game and a given set of demand functions $D$, let

$$
\mathscr{M}_{D}=\left\{x \text { in } A \mid d_{i j}(x)=0 \text { for all } d_{i j} \text { in } D\right\}
$$

be called the bargaining set with respect to $D$.

$\mathscr{M}_{D}$ is just the set of $x$ for which no player has a nonzero demand against another player and it will be shown that under suitable choices of $D, \mathscr{M}_{D}$ can be made into the bargaining set $\mathscr{M}_{1}^{i}$ or the kernel.

If $y$ results from $x$ by a $D$-bounded transfer of size $\alpha$, we say that it is a $D$ bounded transfer of maximal size if there is no $z$ in $A$ and $\beta>\alpha$ such that $z$ results from $x$ by a $D$-bounded transfer of size $\beta$. When $D$ is a set of demand functions, a maximal size transfer is achieved by choosing $(i j)$ to maximize $d_{i j}(x)$ and then transferring $d_{i j}(x)$ from $j$ to $i$. We can now state some conditions under which a series of transfers will converge to $\mathscr{M}_{D}$.

THEOREM 2. Let $D=\left\{d_{i j}\right\}$ be a set of lower semicontinuous demand functions on game $(N, v)$ such that all $D$-bounded transfers are $K$-transfers. If $\left\{x^{i}\right\}$ for $i \geqq 0$ is a sequence of points in $A$ such that each $x^{i+1}$ results from $x^{i}$ by a $D$-bounded transfer of size $\alpha^{i}$ and if an infinite subset of the $\alpha^{i}$ are maximal, then the sequence has a single limit point $\bar{x}$ and $\bar{x}$ is in $\mathscr{M}_{D}$.

Proof. There is a real number $R$ such that $\sum_{1}^{\infty} \alpha^{i}=R$ because the partial sums are monotone increasing and bounded by the $\beta$ of Theorem 1. This implies that the sequence $\left\{x^{i}\right\}$ has a single limit point $\bar{x}$ because $A$ is compact and because a sequence approaching two points would require $\sum \alpha^{i}=\infty$ in order to oscillate between disjoint neighborhoods of those points. Suppose that for some $a$ and $b, d_{a b}(\bar{x})>0$. Choose a $\delta$ such that $0<\delta<d_{a b}(\bar{x})$. Because $d_{a b}$ is continuous, there is an $\varepsilon>0$ such that

$$
\sum_{j \in N}\left|x_{j}-\bar{x}_{j}\right| \leqq \varepsilon \text { implies } d_{a b}(x) \geqq \delta .
$$

Let $r$ be such that $\sum_{r}^{\infty} \alpha^{i}<\min (\varepsilon, \delta)$. It follows that for $i \geqq r$,

$$
\sum_{j \in N}\left|x_{j}^{i}-\bar{x}_{j}\right|<\varepsilon \text { and } \alpha^{i}<\delta \text {. }
$$


These inequalities imply by (28) that $\alpha^{i}<d_{a b}\left(x^{i}\right)$ and so none of the $\alpha^{i}$ for $i>r$ are maximal. This contradicts the assumption that an infinite subset of the $\alpha^{i}$ are maximal and the theorem is proved.

It should be noted that the maximality condition of the theorem can be replaced by any condition which states roughly that arbitrarily small transfers are not always made when much larger ones are possible. Also, convergence is assured even if the transfers are $K$-transfers instead of merely being $D$-bounded. Thus, even if negotiators' transfers crudely approximate the amounts suggested by the $d_{i j}$, convergence is ensured if they stick to $K$-transfers and will approach $\mathscr{M}_{D}$ if they do not always neglect the larger demands.

Applications to the kernel. The kernel $\mathscr{K}$ of a game $(N, v)$ is the set of points $x$ in $A$ such that for all distinct $i$ and $j$ in $N$,

$$
s_{i j}(x) \geqq s_{j i}(x) \text { or } x_{i}=0 .
$$

Set $\mathscr{K}$ is sometimes ([2] and [3]) called the kernel with respect to coalition structure $N$. Other coalition structures are discussed later. The kernel is related to the previous concepts by the following result:

THEOREM 3. For each ordered pair of distinct players $i$ and $j$, let

$$
\begin{aligned}
k_{i j}(x) & =\min \left(\left(s_{i j}(x)-s_{j i}(x)\right) / 2, x_{j}\right) & & \text { if } s_{i j}(x) \geqq s_{j i}(x) \\
& =0 & & \text { otherwise. }
\end{aligned}
$$

Let $K=\left\{k_{i}\right\}$. Then

(i) the $k_{i j}$ are demand functions;

(ii) transfers are $K$-bounded if and only if they are $K$-transfers;

(iii) $\mathscr{M}_{K}$ is the kernel;

(iv) set $K$ satisfies Theorem 2.

Proof. By definition, $k_{i j}$ satisfies (27) and (i) is proven.

If $y$ results from $x$ by a transfer of size $\alpha>0$, (7) is equivalent to

$$
\alpha \leqq\left(s_{a b}(x)-s_{b a}(x)\right) / 2
$$

because of (8) and (9) and so (30) is necessary and sufficient that $y$ results from a $K$-transfer. As discussed before, (27) is necessary and sufficient that there be any transfer of size $\alpha$ from $b$ to $a$. Thus (27) and (30) combine into the condition $\alpha \leqq k_{a b}(x)$ which is necessary and sufficient that there be a $K$-transfer of size $\alpha$ from $b$ to $a$ and (ii) is proven.

The condition $k_{j i}(x)=0$ and condition (30) are both equivalent to the condition that at least one of the three following hold:

$$
\begin{aligned}
s_{i j}(x) & >s_{j i}(x), \\
s_{i j}(x) & \left.=s_{j i}(x) \quad \text { [i.e. }\left(s_{j i}(x)-s_{i j}(x)\right) / 2=0\right], \\
x_{i} & =0 .
\end{aligned}
$$


Part (iii) follows. The demand functions $k_{i j}$ are obviously continuous and because of (iii), Theorem 2 is satisfied and (iv) is proven.

Part (ii) of the theorem explains the notation of previous sections: " $K$-transfers" are now seen to be the same thing as " $K$-bounded transfers." Part (iii) says that $K$ is a set of demand functions which determine the kernel. It follows that any $\mathscr{M}_{D}$ satisfying Theorem 2 must contain the kernel.

A method finding a point in $\mathscr{K}$ can now be stated due to part (iv) and Theorem 2. Given a point $x$ in $A$, compute the various $k_{i j}(x)$ and pick an $(a b)$ such that $k_{a b}(x)$ is greater than or equal to any other $k_{i j}(x)$. Transfer amount $k_{a b}(x)$ from $b$ to $a$. The result is a new vector $y$ in $A$. By repeating this process on $y$ and the succeeding resulting vectors, a sequence of vectors is obtained which converges to the kernel. This scheme was first postulated by M. Maschler at the 1965 Jerusalem Conference on Game Theory where the convergence was given as an open problem. There are, however, some undesirable properties of this method regarding the speed of convergence and knowing when one is close to $\mathscr{K}$. These are best brought out by a couple of examples.

EXAMPLE 1. For $\varepsilon>0$, consider the game defined over $N=\{1,2,3,4,5\}$ by the characteristic function $v$ where:

$v(S)=1$ if $S$ has 4 or 5 elements;

$v(124)=v(134)=v(234)=v(125)=v(235)=1$;

$v(135)=1-\varepsilon$

$v(S)=0$ otherwise.

The kernel of this game (and also the bargaining set) is the single point $(1 / 3,1 / 3$, $1 / 3,0,0)$. The maximal $K$-demand at point $x=(0,0,0,1 / 2,1 / 2)$ is $\varepsilon$, the value of $k_{25}(x)$. Thus one cannot tell from $n$ and the maximal demand how close one is to $\mathscr{K}$. Furthermore, the smaller the value of $\varepsilon$, the larger the number of transfers required to go from $x$ to a point close to $\mathscr{K}$, and so the number of $K$-transfers required to get within $\Delta$ of $\mathscr{K}$ cannot be bounded by a function of $n, \Delta$, and the maximal transfer.

When $\varepsilon=0$, point $x$ is in both the kernel and the bargaining set. Thus the situation may be understood in terms of the discontinuity of the kernel and bargaining set as a function of $v$. Points near $x$ for small $\varepsilon>0$ are far from the kernel, but they have small demands because they are near the kernel of a nearby game.

EXAMPLE 2. Consider the six-person weighted majority game $[16 ; 2,4,4$, $5,6,7]$. The kernel of this game consists of two points, $p_{1}=(0,0,0,0,1 / 2,1 / 2)$ and $p_{2}=(0,1 / 5,1 / 5,1 / 5,1 / 5,1 / 5)$. (This kernel was discovered by A. Kopelowitz at the Hebrew University using a computer.) For $\varepsilon \leqq 1 / 6$, let

$$
x(\varepsilon)=(\varepsilon, \varepsilon, \varepsilon, \varepsilon, 1 / 2-2 \varepsilon, 1 / 2-2 \varepsilon)
$$

The maximal $K$-demand for $x(\varepsilon)$ is $\varepsilon$, yet the transfer sequences beginning at $x(\varepsilon)$ for $\varepsilon>0$ always converge to point $p_{2}$ instead of $p_{1}(=x(0))$. (This is because all the $s_{i j}\left(p_{1}\right)$ are $1 / 2$ whereas some $s_{i j}$ are $1 / 2$ and some are less at $x(\varepsilon)$. Lemma 4 then prevents convergence to $p_{1}$.) The number of transfers required to get from $x(\varepsilon)$ to 
the limit point $p_{2}$ can be made arbitrarily large by taking $\varepsilon$ small enough. Thus even for a fixed game, one cannot, as a function of the maximal demand, bound the number of transfers required to get within $\Delta$ of the limit.

In spite of the worst case possibilities just discussed, this method programmed on a GE-265 time-shared computer has proved to be quite useful in finding kernel points and convergence has generally been very satisfactory. If necessary, algebraic verification that a point $x$ with very small maximal demand is near $\mathscr{K}$ can usually be obtained using the profiles of Maschler and Peleg [3]. Those coalitions which determine the values of the $s_{i j}(x)$ indicate which profile one should try to solve.

Application to the bargaining set. We let symbols such as $x_{S}$ for $S \subseteq N$ represent real vectors indexed over subscripts from set $S$.

An objection to a vector $x_{N}$ in $E$ by player $i$ over player $j$ is a vector $y_{S}$ such that:

$$
\begin{aligned}
i & \in S \text { and } j \notin S ; \\
y_{k} & \geqq x_{k} \quad \text { for all } k \text { in } S ; \\
y_{i} & >x_{i} ; \\
\sum_{S} y_{l} & \leqq v(S) .
\end{aligned}
$$

A counter-objection to an objection $y_{S}$ to $x_{N}$ by $i$ over $j$ is a vector $z_{T}$ such that:

$$
\begin{array}{rlrl}
j & \in T \text { and } i \notin T ; \\
z_{k} & \geqq x_{k} \quad \text { for all } k \text { in } T ; \\
z_{k} & \geqq y_{k} \quad \text { for all } k \text { in } S \cap T ; \\
\sum_{T} z_{k} & \leqq v(T) . &
\end{array}
$$

The bargaining set $\mathscr{M}$ for game $(N, v)$ is the set of all $x$ in $A$ such that each objection to $x$ has a counter-objection. Set $\mathscr{M}$ is often called ([1], [4], and [5]) the bargaining set $\mathscr{M}_{1}^{(i)}$ with respect to coalition structure $N$. Other coalition structures are considered in the next section.

Given distinct players $a$ and $b$ in $N$, let $\mathscr{M}_{a b}$ be the set of all $x$ in $A$ such that every objection by player $a$ over player $b$ has a counter-objection.

Lemma 6. Set $\mathscr{M}_{a b}$ is closed.

Proof. Suppose that $x$ is a limit of $\left\{x^{r}\right\}$ in $\mathscr{M}_{a b}$. The compactness of $A$ insures that $x$ is in $A$. Suppose that $y_{S}$ is an objection to $x$ by $a$ over $b$. Let $y_{S}^{\prime}$ be given by

$$
\begin{aligned}
& y_{i}^{\prime}=y_{i}+\left(y_{a}-x_{a}\right) / n \quad \text { for } i \text { in } S-\{a\}, \\
& y_{a}^{\prime}=x_{a}+\left(y_{a}-x_{a}\right) / n \text {. }
\end{aligned}
$$

Equation (33) says that $\left(y_{a}-x_{a}\right)$ is positive and it follows that $\sum_{s} y_{i}^{\prime}<\sum_{s} y_{i}$ and $y_{S}^{\prime}$ satisfies (34). Thus $y_{S}^{\prime}$ is obviously an objection by $a$ over $b$ to each $x^{-r}$ for $r$ greater 
than some $\beta$. Vector $y_{S}^{\prime}$ must have a counter-objection $z_{T_{r}}^{r}$ for each of these $x^{r}$ and an infinite subset of the $z_{T_{r}}^{r}$ have the same $T_{r}=T$. This subset has a limit $z_{T}$ which is a counter-objection to $y_{S}^{\prime}$ regarded as an objection to $x$. Since $y_{i}^{\prime}>y_{i}$ for $i$ in $S-a, z_{T}$ is also a counter-objection to $y_{S}$ and $x$ must be in $\mathscr{M}_{a b}$.

LEMMA 7. Given distinct $a$ and $b$ in $N$ and an $x$ in $A$, there is a minimal $\alpha \geqq 0$ such that there is a point $y$ in $\mathscr{M}_{a b}$ which results from $x$ by a transfer of size $\alpha$ from $b$ to $a$.

Proof. The $y$ given by $\alpha=x_{b}$ is obviously in $\mathscr{M}_{a b}$ since it is in $A$ and vector $z_{\{b\}}=0$ is a counter-objection to any objection to $y$ by $a$ over $b$. Since $\mathscr{M}_{a b}$ is closed, there must be a minimal $\alpha$.

The last two lemmas are also proved in [4]. We can now state the main result of this section.

THEOREM 4. For distinct $a$ and $b$ in $N$ and $x$ in $A$, let $m_{a b}(x)$ be the $\alpha$ of Lemma 7. Let $M=\left\{m_{i j}\right\}$. Then

(i) the $m_{i j}$ are demand functions;

(ii) $\mathscr{M}_{M}$ is the bargaining set;

(iii) set $M$ satisfies Theorem 2.

Proof. Statements (i) and (ii) are obvious from Lemma 7. If $s_{j i}(x) \geqq s_{i j}(x)$, it is well known that any objection $y$ to $x$ by $i$ over $j$ has a counter-objection. (The larger or equal excess of $j$ over $i$ insures that any increases $\left(y_{k}-x_{k}\right)$ for $k$ in $S$ can be matched and a counter-objection sustained [2].) Thus $M$-bounded transfers are all $K$-transfers. To prove the lower semicontinuity of $m_{i j}$, let $x^{k}$ be a sequence of points that converge to $x$, let $\alpha^{k}=m_{i j}\left(x^{k}\right)$, and let $y^{k}$ be the points in $\mathscr{M}_{i j}$ such that $y^{k}$ results from $x^{k}$ by a transfer of size $\alpha^{k}$ from $j$ to $i$. If $\alpha$ is any limit point of the $\alpha^{k}$, the corresponding subsequence of the $y^{k}$ obviously converges to the point $y$ which results from $x$ by a transfer of size $\alpha$ from $j$ to $i$. This point is in $\mathscr{M}_{i j}$ because $\mathscr{M}_{i j}$ is compact, hence $m_{i j}(x) \leqq \alpha$ and $m_{i j}$ must be lower semicontinuous.

The $m_{i j}$ are actually continuous. We now know from Theorem 2 that any sequence of maximal $M$-bounded transfers converges to the bargaining set. This convergence has the undesirable properties mentioned in Example 1 since the bargaining set and the kernel are the same for that example.

Other coalition structures. The previous results can be applied to other coalition structures in a rather straightforward manner. Given a coalition structure (i.e. partition) $\mathscr{B}=\left\{B_{1}, \ldots, B_{m}\right\}$, we replace set $A$ by

$$
A^{\prime}=\left\{x \mid x_{i} \geqq 0 \text { for } i \text { in } N \text { and } \sum_{B} x_{i}=v(B) \text { for } B \text { in } \mathscr{B}\right\} \text {. }
$$

Equations (12) and (13) require a scalar change if $v(B)>1$, the same as they would under change of normalization, but they remain sufficient to prove Theorem 1 . The demand functions have to be defined with respect to $\mathscr{B}$ and require the additional condition $d_{i j}(x)=0$ whenever $i$ and $j$ are not in the same $B$ of $\mathscr{B}$. This condition 
together with (27) insures that there is always a $D$-bounded transfer for all $\alpha \leqq d_{a b}(x)$, and Theorem 2 holds. Appropriate versions of Theorems 3 and 4 follow.

These results give still another proof that the kernel and/or the bargaining set are always nonempty for each coalition structure. Previous proofs appear in [2], [3], [4], and [5].

Acknowledgement. The author is grateful to M. Maschler of the Hebrew University and L. J. Billera of Cornell University for critical readings of this manuscript.

\section{REFERENCES}

1. R. J. Aumann and M. Maschler, "The bargaining set for cooperative games," pp. 443-476, in Advances in game theory, Ann. of Math. Studies No. 52, Princeton Univ. Press, Princeton, N. J., 1964.

2. M. Davis and M. Maschler, The kernel of a cooperative game, Naval Res. Logist. Quart. 12 (1965), 223-259.

3. M. Maschler and B. Peleg, A characterization, existence proof and dimension bounds for the kernel of a game, Pacific J. Math. 18 (1966), 289-328.

4. M. Davis and M. Maschler, "Existence of stable payoff configurations for cooperative games," pp. 39-52, in Essays in mathematical economics in honor of Oscar Morgenstern, Princeton Univ. Press, Princeton, N. J., 1967. (A short form of this paper appears in Bull. Amer. Math. Soc. 69 (1963), 106-108.)

5. B. Peleg, "Existence theorem for the bargaining set $\mathscr{K}_{1}^{(1)}$," pp. 53-56, in Essays in mathematical economics in honor of Oscar Morgenstern, Princeton Univ. Press, Princeton, N. J., 1967. (A short form of this paper appears in Bull. Amer. Math. Soc. 69 (1963), 109-110.)

General Electric Research and Development Center, SCHENECTADY, NeW YORK 